\title{
Cardiovascular Functions and Dopamine: Mechanism of Action in Adult Male Anesthetized Balady Rabbits
}

\author{
Enas A. Hamed, ${ }^{1}$ Emtethal M Moustafa, ${ }^{1}$ Nashwa A Abd El-Mottalib ${ }^{1}$ \\ ${ }^{1}$ Department of Physiology, Faculty of Medicine, Assiut University, \\ Assiut, Egypt
}

\begin{abstract}
This study aimed to elucidate mechanism(s) that mediate dopamine regulation of cardiovascular system (CVS) functions. Forty eight adult male anesthetized Balady rabbits (4 experiments, 8 groups, 6 animals each) were included. Experiment I assessed the effect of intravenous (iv) dopamine infusion (0.1, 1, 4 and $12 \mu \mathrm{g} / \mathrm{kg} / \mathrm{min}$ ) on diastolic $(D B P)$, systolic $(S B P)$, mean blood pressure $(M B P)$, heart rate $(H R)$, cardiac contractility (CC) and renal sympathetic nerve activity (RSNA). Experiment II assessed the effect of dopamine infusion on ventricular sarcomere length. Experiment III confirmed the contribution of dopamine receptor subtype(s). Experiment IV evaluated adrenergic receptors involved in dopamine's action. Mean $B P, C C, H R$ and RSNA were recorded by physiograph. At low dopamine infusion rate $D B P, M B P, C C$ and RSNA were decreased; while sarcomere length and A:I ratio were increased. At high dopamine infusion rate $D B P, S B P, M B P, H R$ and $C C$ were increased while; sarcomere length and A:I ratio were decreased. $D_{1}$-like receptor activation decreased $M B P$; while $D_{2}$-like receptor activation decreased $M B P, C C$, and RSNA. Both $D_{1}$-and $D_{2}$-like receptors blockade attenuated hypotensive response, whereas $C C$ was abolished by $D_{2}$ receptor blockade. Mean BP, HR and CC were not changed after $D_{I^{-}}$and $D_{2}$-like receptors blockade, but decreased after $D_{I^{-}}$and $D_{2}$ like receptors activation. Low dopamine infusion into animals pre-treated with $\alpha$ adrenoceptor blockade (reserpine) or $\beta$-adrenoceptor blockade (propranolol) decreased MBP and CC whereas, with high dopamine infusion, the HR and CC were increased after $\alpha$-adrenoceptor blockade and MBP was increased after $\beta$ adrenoceptor blockade. From this study, we can conclude that dopamine elicits biphasic effect on CVS. Low dopamine doses acts via stimulation of $D_{1^{-}}$and $D_{2^{-}}$like receptors. With increasing dose, actions occur via stimulation of $\alpha$-and $\beta$-adrenergic receptors. Normal endogenous dopamine may not alter basal cardiovascular functions.
\end{abstract}

\section{INTRODUCTION}

Dopamine (DA) is an endogenous catecholamine that serves not only as a precursor for norepinephrine and epinephrine but also as a neurotransmitter. Dopamine is involved in a wide variety of physiological processes both in central nervous system (CNS) and in peripheral tissues and organs ${ }^{(1)}$. In the mammalian brain, DA controls a variety of functions including locomotors activity, cognition, food intake and endocrine regulation. This catecholamine also plays multiple 
roles in the periphery as a modulator of cardiovascular functions (CVS), catecholamine release, hormone secretions, vascular tone, renal functions and gastrointestinal motilities ${ }^{(2)}$.

Dopamine exerts its physiological actions via $\alpha$ - and $\beta$-adrenergic receptors as well as via specific dopaminergic receptors, a class of cell surface receptors coupled to Gproteins. Dopaminergic receptors have been classified into two families ( $\mathrm{D}_{1}$-like and $\mathrm{D}_{2}$-like) based on biochemical, pharmacological and molecular cloning studies. In mammals, two $\mathrm{D}_{1}$-like receptors $\left(\mathrm{D}_{1}\right.$ and $\mathrm{D}_{5}$ ) have been cloned and linked to stimulation of adenyl cyclase stimulate phospholipase $\mathrm{C}^{(3)}$. Three $\mathrm{D}_{2}$-like receptors have been cloned $\left(D_{2}, D_{3}\right.$ and $\left.D_{4}\right)$ and linked to inhibition of adenyl cyclase ${ }^{(2)}$. All the mammalian dopamine receptors initially cloned from the brain, have been found to be expressed outside $\mathrm{CNS}$, in such sites as the heart, blood vessels, carotid body, kidney, adrenal gland, parathyroid gland, gastrointestinal tract, vascular smooth muscle, and on the terminals of postganglionic sympathetic nerves $(4,5,6)$.

It is evident from forgoing studies on the multiple dopamine receptors that activation and /or blockade of them can lead to pronounced changes in cardiovascular functions. Indeed, rodents with genetic hypertension and human with essential hypertension are associated with a defective dopamine production and/or dopamine receptor functions ${ }^{(2,7)}$. Moreover, dopamine receptor blockade is associated with the development of hypertension in saline-loaded rats. Finally, inhibition of dopamine synthesis outside CNS accelerates hypertension development in spontaneously hypertensive rats ${ }^{(8)}$.

The present study was designed to determine the possible receptor subtype(s) which mediate the effect of dopamine in regulation of some cardiovascular functions as arterial blood pressure (ABP), heart rate (HR) and cardiac contractility (CC). Also, to study the possible association between dopamine and sympathetic nervous system on affecting cardiovascular functions.

\section{MATERIALS \& METHODS}

\section{Animals.}

This investigation was carried out on 48 adult male Balady rabbits, weighing $(1.2-1.5 \mathrm{~kg})$ divided into 8 groups, 6 animals each. On arrival, animals were caged individually in room temperature with natural lightdark cycle with free access to water and commercial rabbit food. This study was carried out on 4 experiments at the same period each day (8-10 am). After one week of acclimatization, rabbits were randomly assigned into the studies. After termination of the experimental protocols, all animals were scarified. The surgical and experimental procedures were carried out according to "Guideline on Experiments on Animal" at Faculty of Medicine, Assiut University and approved by Ethical Committee.

Reagents.

Dopamine (3-hydroxytyramine hydrochloride) was purchased from Fluka Company [Buchs, Switzerland]; reserpine, $\mathrm{SCH}-23390$ and metochlopramide from Sigma Aldrich [Seelze, Germany]; urethane from Aldrich Chemical Company [Wis., USA]; heparin from Pharmacia 
Pharmacuticals [Cairo, Egypt]; SKF38393 and bromocriptine from ICN Biomedicals Inc. [Ohio, USA] and propranolol from Macclesfeild [Cheshire, UK].

\section{Surgical Preparation.}

The surgical technique was done according to steps described by Muhlbauer et al. (9). Rabbits were anesthetized through intraperitoneal (ip) injection of urethane $(600 \mathrm{mg} / \mathrm{kg})$. The trachea was exposed by blunt dissection and intubated with a short cannula to insure free airways during experiments. One catheter was inserted into right jugular vein for iv infusion of the drugs via its connection to the syringe pump (ColeParmer, 74900-00-05, Cole-Parmer Instrument Company, USA) and another catheter into left common carotid artery for ABP recording and blood sampling. Heparin $(0.25 \mathrm{ml})$ was injected iv through the catheter to prevent intracatheter clotting. Following surgical preparation, animals were allowed to reach steady state condition defined by stable ABP and HR which achieved within 15 min.

\section{Study protocol.}

In Experiment I, two groups of rabbits (Groups 1 and 2) were used. After the steady state conditions reached, a baseline period (control) of 30 min was performed. During the control period the animals received iv infusion of $0.9 \%$ sterile sodium chloride solution (vehicle) at a rate of $0.02 \mathrm{ml} / \mathrm{min}$ delivering approximately $0.6 \mathrm{ml}$ solution throughout the experiment. After completion of baseline period, infusion was switched to dopamine in 4 doses. Group (1) received iv infusion of dopamine at a rate of 0.1 and $1 \mu \mathrm{g} / \mathrm{kg} / \mathrm{min}$ with 15 min interval delivering approximately
$0.6 \mathrm{ml}(0.02 \mathrm{ml} / \mathrm{min})$ throughout the experiment for each dose. Group (2) received iv infusion of dopamine at a rate of 4 and $12 \mu \mathrm{g} / \mathrm{kg} / \mathrm{min}$ with 15 min interval delivering approximately $0.6 \mathrm{ml}(0.02 \mathrm{ml} / \mathrm{min})$ throughout the experiment for each dose. Arterial BP, $\mathrm{HR}$ and $\mathrm{CC}$ were recorded before (baseline), at 1, 15 and 30 min during dopamine infusion (experimental period) and at 15 min after termination of infusion (recovery period). Renal sympathetic nerve activity (RSNA) was recorded before (baseline) and at $30 \mathrm{~min}$ during dopamine infusion at rates of 1 and 12 $\mu \mathrm{g} / \mathrm{kg} / \mathrm{min}$ (preferred effective low and high doses respectively).

In experiment II, 3 groups of rabbits (Groups 3-5) were used. Group 3 (control group) received iv infusion of normal saline at a rate of $0.02 \mathrm{ml} / \mathrm{min}$ for $30 \mathrm{~min}$; groups (4 and 5) received iv infusion of dopamine at a rate of 1 and $12 \mu \mathrm{g} / \mathrm{kg} / \mathrm{min}$ for 30 min successively. After infusion termination, specimens from left ventricle were taken and prepared for electro-microscopic examination. Sarcomere length $(\mu \mathrm{m})$ and ratio of $\mathrm{A}$ : I band were measured.

In experiment III, two groups (6 and 7) of rabbits were used. Animals of group (6) received iv infusion of SKF-3839 (5 $\mu \mathrm{g} / \mathrm{kg} / \mathrm{min}$ for $15 \mathrm{~min})$, a selective agonist of $\mathrm{D}_{1}$-like receptor (10). After a rest period of $15 \mathrm{~min}$, the same animals received iv infusion of $1 \mu \mathrm{g} / \mathrm{kg} / \mathrm{min}$ dopamine $20 \mathrm{~min}$ after a previous administration of $\mathrm{SCH}$ $23390(50 \mu \mathrm{g} / \mathrm{kg}$, ip), a selective antagonist of $\mathrm{D}_{1}$-like receptor ${ }^{(\mathbf{1 1})}$. After an additional 15 min rest period, rabbits received iv infusion of 12 $\mu \mathrm{g} / \mathrm{kg} / \mathrm{min}$ dopamine $20 \mathrm{~min}$ after previous administration of $\mathrm{SCH}-$ $23390(50 \mu \mathrm{g} / \mathrm{kg}$, ip). Animals of 
group (7) received iv infusion of bromocriptine $(1 \mu \mathrm{g} / \mathrm{kg} / \mathrm{min}$ for 15 min), a selective agonist of $\mathrm{D}_{2}$-like receptor $^{(\mathbf{1 2 )}}$. After a rest period of 15 min, the same animals received iv infusion of $1 \mu \mathrm{g} / \mathrm{kg} / \mathrm{min}$ dopamine 20 min after a previous administration of metoclorpramide (15 mg/kg, ip), a selective antagonist of $\mathrm{D}_{2}$-like receptor $^{(13)}$. After an additional $15 \mathrm{~min}$ rest period, rabbits received iv infusion of $12 \mu \mathrm{g} / \mathrm{kg} / \mathrm{min}$ dopamine 20 min after previous administration of metoclorpramide (15 mg/kg, ip). Arterial BP, HR, CC and RSNA were recorded before (baseline) and $15 \mathrm{~min}$ during $\mathrm{D}_{1}{ }^{-}$and $\mathrm{D}_{2}$-like receptor agonist and antagonism treatment.

In experiment $I V$, one group (Group 8) was used. Animals of this group received iv infusion of 1 and 12 $\mu \mathrm{g} / \mathrm{kg} / \mathrm{min}$ dopamine for $15 \mathrm{~min}$ with 15 min interval, 20 min after previous administration of reserpine $(\alpha-$ adrenoceptor blockade, $3 \mathrm{mg} / \mathrm{kg}$, ip) ${ }^{(\mathbf{1 4})}$. After a rest period of $15 \mathrm{~min}$, the same animals received iv infusion of 1 and $12 \mu \mathrm{g} / \mathrm{kg} / \mathrm{min}$ dopamine for 15 min with 15 min interval, $20 \mathrm{~min}$ after previous administration of propranolol ( $\beta$-adrenoceptor blockade, $1 \mathrm{mg} / \mathrm{kg}, \mathrm{sc})^{(\mathbf{1 5})}$. Arterial BP, HR and $\mathrm{CC}$ were recorded before (control) and at 15 min during dopamine infusion.

Arterial BP was recorded by EM 75/No 4403" pressure transducer that convert ABP to a proportional electrical signals. Signals were amplified using "Strain Gauge Coupler Fc 137" of physiograph [Harvard Apparatus Limited, 50-8622, Kent, UK]. Systolic (SBP) and diastolic blood pressure (DBP) of the animal were recorded. Mean BP was calculated as DBP $+[1 / 3(\mathrm{SBP}-$ $\mathrm{DBP})]^{(\mathbf{1 6})}$. Electrocardiogram (ECG) was recorded using $\left[\begin{array}{ll}F x & 2111\end{array}\right.$ Electrocardiograph Cardimax]. HR was registered with "Fc123 ECG coupler" connected to the physiograph. Heart isometric contractility was monitored by means of a photoelectric force transducer [E \& M Physiograph Myograph, Houston TX, USA] connected to a heart clips at apex of the ventricles. The amplitude of contractions in $\mathrm{mm}$ is used as an index of cardiac contractility. Renal nerve traffics were recorded after their stimulation with micro electrodes connected to stimulator cable [electronic stimulator, SEN 3201]. Renal nerve signals were magnified with Harvard coupler apparatus [50-6360]. Renal SNA was recorded according to the method described by Thoren \& Ricksten $^{(17)}$. The amplitude of bursts $(\mathrm{mm})$ and frequency (rate/min) were used as an index of sympathetic nerve activity. Samples from ventricular tissues of the rabbits were prepared for electro-microscopic examination.

Statistical analysis.

The statistical analysis was performed using Prism software version 3. Data were expressed as mean +/- standard error (SE). For inter-group comparison, analysis with two-sided paired Student's " $t$ " test or one way ANOVA test was carried out as appropriate. Differences between results were considered statistically significant when $P$ - value was less than 0.05 .

\section{RESULTS}

Tables (1) and Figures (1 and 2) summarize the effect of intravenous dopamine infusion $(0.1,1,4$ and 12 $\mu \mathrm{g} / \mathrm{kg} / \mathrm{min})$ on ABP $(\mathrm{mmHg})$, HR (beat $/ \mathrm{min}$ ) and $\mathrm{CC}(\mathrm{mm})$ respectively in adult male rabbits. From these 
tables it is clear that at dose 1 $\mu \mathrm{g} / \mathrm{kg} / \mathrm{min}$, a significant decrease was observed in DBP $(P<0.05, P<0.01$ and $P<0.01)$ and MBP $(P<0.05, P$ $<0.001$ and $P<0.001)$ compared with baseline level at 1,15 and $30 \mathrm{~min}$ during infusion respectively. While, at dose $12 \mu \mathrm{g} / \mathrm{kg} / \mathrm{min}$, a significant increase was observed in DBP $(P$ $<0.05)$, SBP $(P<0.01)$, and $\mathrm{MBP}(P$ $<0.01, \quad P<0.001$ and $P<0.001)$ compared with baseline level at 1,15 and $30 \mathrm{~min}$ during infusion successively.

Heart rate was significantly increased at 15 and 30 min during iv dopamine infusion at doses of 4 $\mu \mathrm{g} / \mathrm{kg} / \mathrm{min} \quad(P<0.05)$ and 12 $\mu \mathrm{g} / \mathrm{kg} / \mathrm{min}(P<0.05)$ (Table 2; Figures 1 and 2). Cardiac contractility at 1, 15 and 30 min during dopamine infusion showed a significant decrease with a dose of $1 \mu \mathrm{g} / \mathrm{kg} / \mathrm{min} \quad(P<0.05, P$ $<0.01$ and $P<0.01$ respectively) but a significant increase was observed with a dose of $12 \mu \mathrm{g} / \mathrm{kg} / \mathrm{min}(P<0.05, P$ $<0.01$ and $P<0.01$ successively). Whereas, at a dose of $4 \mu \mathrm{g} / \mathrm{kg} / \mathrm{min}$, a significant increase in $\mathrm{CC}$ was observed at 15 and $30 \mathrm{~min}$ only ( $P$ $<0.05$ ) (Table 3; Figures 1 and 2).

Intravenous infusion of dopamine at a dose of $1 \mu \mathrm{g} / \mathrm{kg} / \mathrm{min}$ led to a significant decrease in frequency and amplitude $(P<0.001)$ of RSNA and a significant increase in sarcomere length and A:I ratio $(P<0.01)$. While, iv infusion of dopamine at a dose of $12 \mu \mathrm{g} / \mathrm{kg} / \mathrm{min}$ led a to decrease in sarcomere length and A:I ratio ( $P$ $<0.05$ ) (Table 4, Figures 3-6).

Activation of $D_{1}$-like receptor by intravenous SKF-38393 infusion resulted in a significant decrease in MBP $(P<0.01)$. Whereas, activation of $\mathrm{D}_{2}$ receptors by iv bromocriptine infusion resulted in a significant decrease in $\mathrm{MBP}, \mathrm{CC}$ and frequency and amplitude of RSNA $(P<0.05, P$ $<0.01, \quad P<0.001$ and $P<0.001$ respectively) (Table 5).

Administration of $\mathrm{D}_{1}$-like antagonist SCH-23390 alone didn't cause any significant change in the levels of MBP, HR and CC compared with baseline levels. Intravenous dopamine infusion after SCH-23390 pre-treatment at a dose of $1 \mu \mathrm{g} / \mathrm{kg} / \mathrm{min}$ led to a significant decrease in the MBP and $\mathrm{CC}(P<0.05, P<0.01)$, meanwhile, at dose $12 \mu \mathrm{g} / \mathrm{kg} / \mathrm{min}$ led to significant increase in MBP, HR and $\mathrm{CC}(P<0.01, P<0.05$ and $P$ $<0.01$ successively) compared to control level. Administration of $\mathrm{D}_{2-}$ like receptor antagonist metoclopramide alone didn't cause any significant change in the levels of MBP, HR and CC compared with baseline levels. Intravenous dopamine infusion after metoclopramide pretreatment led to a significant decrease in the $\operatorname{MBP}(P<0.05)$, while, 12 $\mu \mathrm{g} / \mathrm{kg} / \mathrm{min}$ led to a significant increase in the MBP, HR and CC $(P<0.01)$ compared to control level (Table 6).

After reserpine pre-treatment, iv dopamine infusion at a rate of 1 $\mu \mathrm{g} / \mathrm{kg} / \mathrm{min}$ led to a significant decreased in MBP and CC $(P<0.05$ and $P<0.01$ respectively), while at dose of $12 \mu \mathrm{g} / \mathrm{kg} / \mathrm{min}$ led to significant increased in HR and CC $(P$ $<0.05, \quad P \quad<0.01 \quad$ successively) compared with the pre-injected dopamine (control) levels and after propranolol pre-treatment, iv dopamine infusion at a rate of 1 $\mu \mathrm{g} / \mathrm{kg} / \mathrm{min}$ led to a significant decreased in MBP and CC $(P<0.01)$, while at a dose of $12 \mu \mathrm{g} / \mathrm{kg} / \mathrm{min}$ led to significant increase in MBP $(P<0.05)$ compared to control level (Table 7). 
Table (1): The effect of intravenous dopamine infusion $(0.1,1,4,12 \mu \mathrm{g} / \mathrm{kg} / \mathrm{min})$ on arterial blood pressure $(\mathrm{mmHg})$ in adult male rabbits.

\begin{tabular}{|c|c|c|c|c|c|c|c|c|c|c|c|c|c|c|c|c|}
\hline \multirow{3}{*}{$\stackrel{\leftrightarrow}{\circ}$} & \multirow{3}{*}{ Variables } & \multicolumn{5}{|c|}{ Diastolic Pressure (mmHg) } & \multicolumn{5}{|c|}{ Systolic Pressure ( $\mathrm{mmHg})$} & \multicolumn{5}{|c|}{ Mean Blood Pressure ( $\mathrm{mmHg}$ ) } \\
\hline & & \multirow{2}{*}{ Base } & \multicolumn{3}{|c|}{ Time (min) } & \multirow{2}{*}{ Rec. } & \multirow{2}{*}{ Base } & \multicolumn{3}{|c|}{ Time (min) } & \multirow{2}{*}{ Rec. } & \multirow{2}{*}{ Base } & \multicolumn{3}{|c|}{ Time (min) } & \multirow{2}{*}{$\operatorname{Rec}$. } \\
\hline & & & 1 & 15 & 30 & & & 1 & 15 & 30 & & & 1 & 15 & 30 & \\
\hline \multirow{4}{*}{3} & & 10 & 75.17 & 76.50 & 74.00 & 76.67 & 30 & 101.80 & 100.80 & 102.70 & 30 & 1 & 84.06 & 84.61 & 83.56 & 85.56 \\
\hline & & & \pm 2.34 & \pm 2.14 & 00 & \pm 2.77 & & & \pm 3.27 & \pm 1.76 & & 58 & \pm 2.14 & \pm 2.51 & \pm 2.55 & \pm 2.52 \\
\hline & P value & & $P>0.05$ & $P>0.05$ & $P>0.05$ & $P>0.05$ & & $P>0.05$ & $P>0.05$ & $P>0.05$ & $P>0.05$ & & $P>0.05$ & $P>0.05$ & $>0.05$ & $P>0.05$ \\
\hline & \%Change & & $1.09 \downarrow$ & $0.66 \uparrow$ & $2.63 \downarrow$ & $0.88 \uparrow$ & & $1.45 \downarrow$ & $2.42 \downarrow$ & $0.58 \downarrow$ & 0.00 & & $1.23 \downarrow$ & $59 \downarrow$ & $1.82 \downarrow$ & $0.53 \uparrow$ \\
\hline \multirow{4}{*}{-1} & & 74.00 & 63.33 & 52.83 & 58.50 & 78.67 & 105.20 & $104.20^{\circ}$ & $97.50^{-}$ & 97.17 & 100.00 & 84.39 & 76.94 & 67.72 & 71.39 & 85.78 \\
\hline & $\pm \mathrm{SE}$ & \pm 3.65 & \pm 1.52 & \pm 3.83 & \pm 2.75 & \pm 2.62 & \pm 2.59 & \pm 1.72 & \pm 1.71 & \pm 1.64 & \pm 0.52 & \pm 3.21 & \pm 1.35 & \pm 2.84 & \pm 1.86 & \pm 1.63 \\
\hline & Pvalue & & $P<0.05$ & $P<0.01$ & $P<0.01$ & $P>0.05$ & & $P>0.05$ & $P>0.05$ & $P>0.05$ & $P>0.05$ & & $P<0.05$ & $P<0.001$ & $p<0.001$ & $P>0.05$ \\
\hline & \%Change & & $14.42 \downarrow$ & $28.61 \downarrow$ & $20.95 \downarrow$ & $6.31 \uparrow$ & & $0.95 \downarrow$ & $7.32 \downarrow$ & $7.60 \downarrow$ & $4.94 \downarrow$ & & $8.83 \downarrow$ & $19.75 \downarrow$ & $15.40 \downarrow$ & $1.65 \uparrow$ \\
\hline \multirow{4}{*}{$T$} & Mean & 79.33 & 82.67 & 87.83 & 86.17 & 84.67 & 110.00 & $106.30^{\circ}$ & 109.17 & 115.50 & 115.30 & $89 . \overline{56}$ & 90.56 & $95.11^{-1}$ & 95.94 & 94.89 \\
\hline & $\pm \mathrm{SE}$ & \pm 3.71 & \pm 7.51 & \pm 5.78 & \pm 3.66 & \pm 3.49 & \pm 5.16 & \pm 8.01 & \pm 4.52 & \pm 4.11 & \pm 4.22 & \pm 3.56 & \pm 7.48 & \pm 5.26 & \pm 1.79 & \pm 5.04 \\
\hline & P value & & $9>0.05$ & $P>0.05$ & $P>0.05$ & $P>0.05$ & & $P>0.05$ & $P>0.05$ & $P>0.05$ & $P>0.05$ & & $P>0.05$ & $P>0.05$ & $P>0.05$ & $P>0.05$ \\
\hline & \%Change & & $4.21 \uparrow$ & $10.71 \uparrow$ & $8.62 \uparrow$ & $6.73 \uparrow$ & & $3.36 \downarrow$ & $0.75 \downarrow$ & $5.00 \uparrow$ & $4.82 \uparrow$ & & $1.12 \uparrow$ & $6.20 \uparrow$ & $7.12 \uparrow$ & $5.95 \uparrow$ \\
\hline \multirow{4}{*}{ II } & Mean & 79.33 & 90.83 & 94.50 & 94.50 & 86.67 & 110.00 & $128.70^{\circ}$ & $138.70^{-}$ & 136.80 & 116.00 & 89.56 & 103.4 & $109.20^{\circ}$ & $108.60^{-}$ & 96.44 \\
\hline & $\pm \mathrm{SE}$ & \pm 3.33 & \pm 1.94 & \pm 2.68 & \pm 1.89 & \pm 2.62 & \pm 5.63 & \pm 7.67 & \pm 7.33 & \pm 8.04 & \pm 6.22 & \pm 3.06 & \pm 3.43 & \pm 3.69 & \pm 3.26 & \pm 3.35 \\
\hline & P value & & $p<0.05$ & $p<0.05$ & $p<0.05$ & $P>0.05$ & & $p<0.01$ & $p<0.01$ & $p<0.01$ & $P>0.05$ & & $p<0.01$ & $\rho<0.001$ & $0<0.001$ & $>0.05$ \\
\hline & \%Change & & $14.50 \uparrow$ & $19.12 \uparrow$ & $19.12 \uparrow$ & $9.25 \uparrow$ & & $16.97 \uparrow$ & $26.06 \uparrow$ & $24.39 \uparrow$ & $5.45 \uparrow$ & & $15.45 \uparrow$ & $21.93 \uparrow$ & $21.26 \uparrow$ & $7.68 \uparrow$ \\
\hline
\end{tabular}

SE : Standard Error.

$\downarrow$ : Percent decrease, $\uparrow$ : Percent increase.

Base: Baseline. Rec: Recovery

Significance versus base level using one way ANOVA test 
Table (2): The effect of intravenous dopamine infusion $(0.1,1,4,12 \mu \mathrm{g} / \mathrm{kg} / \mathrm{min})$ on heart rate (beat $/ \mathrm{min})$ in adult male rabbits.

\begin{tabular}{|c|c|c|c|c|c|c|}
\hline \multirow{2}{*}{ Dose } & \multirow{2}{*}{ Variables } & \multirow{2}{*}{ Baseline } & \multicolumn{3}{|c|}{ Time (min) } & \multirow[t]{2}{*}{ Recovery } \\
\hline & & & 1 & 15 & 30 & \\
\hline \multirow{3}{*}{$0.1(\mu \mathrm{g} / \mathrm{kg} / \mathrm{min})$} & Mean \pm SE & $285.00 \pm 8.94$ & $285.80 \pm 11.72$ & $280.00 \pm 9.31$ & $281.70 \pm 10.14$ & $283.3 \pm 12.02$ \\
\hline & P value & & $P>0.05$ & $P>0.05$ & $P>0.05$ & $P>0.05$ \\
\hline & $\%$ Change & & $0.28 \downarrow$ & $1.75 \downarrow$ & $1.16 \downarrow$ & $0.60 \downarrow$ \\
\hline \multirow{3}{*}{$1(\mu \mathrm{g} / \mathrm{kg} / \mathrm{min})$} & Mean $\pm \mathrm{SE}$ & $285.80 \pm 8.80$ & $278.30 \pm 11.38$ & $276.70 \pm 7.60$ & $277.50 \pm 9.29$ & $285.00 \pm 7.64$ \\
\hline & $P$ value & & $P>0.05$ & $P>0.05$ & $P>0.05$ & $P>0.05$ \\
\hline & $\%$ Change & & $2.62 \downarrow$ & $3.18 \downarrow$ & $2.90 \downarrow$ & $0.28 \downarrow$ \\
\hline \multirow{3}{*}{$4(\mu \mathrm{g} / \mathrm{kg} / \mathrm{min})$} & Mean $\pm \mathrm{SE}$ & $283.30 \pm 8.43$ & $285.00 \pm 10.88$ & $321.70 \pm 8.33$ & $326.70 \pm 12.56$ & $281.70 \pm 9.10$ \\
\hline & P value & & $P>0.05$ & $P<0.05$ & $p<0.05$ & $P>0.05$ \\
\hline & $\%$ Change & & $0.60 \uparrow$ & $13.55 \uparrow$ & $15.32 \uparrow$ & $0.56 \downarrow$ \\
\hline \multirow{3}{*}{$12(\mu g / \mathrm{kg} / \mathrm{min})$} & Mean $\pm \mathrm{SE}$ & $284.20 \pm 8.98$ & $290.00 \pm 3.65$ & $328.3 \pm 11.95$ & $320.0 \pm 10.33$ & $288.30 \pm 6.01$ \\
\hline & $P$ value & & $P>0.05$ & $P<0.05$ & $P<0.05$ & $P>0.05$ \\
\hline & $\%$ Change & & $2.04 \uparrow$ & $15.54 \uparrow$ & $12.61 \uparrow$ & $1.44 \uparrow$ \\
\hline
\end{tabular}

SE: Standard Error,

$\downarrow$ : Percent decrease, $\uparrow:$ Percent increase.

Significance versus baseline level using one way ANOVA test. 
Table (3): The effect of intravenous dopamine infusion $(0.1,1,4,12 \mu \mathrm{g} / \mathrm{kg} / \mathrm{min})$ on cardiac contractility (mm) in adult male rabbits.

\begin{tabular}{|c|c|c|c|c|c|c|}
\hline \multirow{2}{*}{ Dose } & \multirow{2}{*}{ Variables } & \multirow{2}{*}{ Baseline } & \multicolumn{3}{|c|}{ Experimental Period (min) } & \multirow[t]{2}{*}{ Recovery } \\
\hline & & & 1 & 15 & 30 & \\
\hline \multirow[t]{3}{*}{$0.1(\mu \mathrm{g} / \mathrm{kg} / \mathrm{min})$} & Mean \pm SE & $30.83 \pm 3.71$ & $30.17 \pm 4.21$ & $28.50 \pm 4.16$ & $28.83 \pm 4.36$ & $31.67 \pm 4.21$ \\
\hline & $P$ value & & $P>0.05$ & $P>0.05$ & $P>0.05$ & $P>0.05$ \\
\hline & $\%$ Change & & $2.14 \downarrow$ & $8.18 \downarrow$ & $6.49 \downarrow$ & $2.72 \uparrow$ \\
\hline \multirow{3}{*}{$1(\mu \mathrm{g} / \mathrm{kg} / \mathrm{min})$} & Mean \pm SE & $32.50 \pm 2.90$ & $21.83 \pm 4.58$ & $19.00 \pm 3.11$ & $20.67 \pm 2.70$ & $29.83 \pm 3.50$ \\
\hline & $P$ value & & $P<0.05$ & $P<0.01$ & $P<0.01$ & $P>0.05$ \\
\hline & $\%$ Change & & $32.83 \downarrow$ & $41.54 \downarrow$ & $36.40 \downarrow$ & $8.22 \downarrow$ \\
\hline \multirow{3}{*}{$4(\mu \mathrm{g} / \mathrm{kg} / \mathrm{min})$} & Mean \pm SE & $30.83 \pm 3.56$ & $33.67 \pm 3.78$ & $38.67 \pm 1.89$ & $38.00 \pm 2.13$ & $31.83 \pm 3.65$ \\
\hline & $P$ value & & $P>0.05$ & $P<0.05$ & $P<0.05$ & $P>0.05$ \\
\hline & $\%$ Change & & $9.21 \uparrow$ & $25.43 \uparrow$ & $23.26 \uparrow$ & $3.24 \uparrow$ \\
\hline \multirow{3}{*}{$12(\mu \mathrm{g} / \mathrm{kg} / \mathrm{min})$} & Mean $\pm S E$ & $29.00 \pm 3.54$ & $35.67 \pm 1.98$ & $39.50 \pm 2.55$ & $39.33 \pm 2.96$ & $30.17 \pm 0.75$ \\
\hline & $P$ value & & $P<0.05$ & $P<0.01$ & $P<0.01$ & $P>0.05$ \\
\hline & $\%$ Change & & $23.00 \uparrow$ & $36.21 \uparrow$ & $35.62 \uparrow$ & $4.03 \uparrow$ \\
\hline
\end{tabular}

SE : Standard Error.

$\downarrow$ : Percent decrease, $\uparrow$ : Percent increase.

Significance versus baseline level using one way ANOVA test. 
Table (4): Effect of intravenous dopamine infusion ( 1 and $12 \mu \mathrm{g} / \mathrm{kg} / \mathrm{min}$ ) on sympathetic nerve activity, sarcomere length and A:I ratio in adult male rabbits.

\begin{tabular}{|c|c|c|c|c|c|c|c|c|c|c|c|c|}
\hline \multirow{4}{*}{$\begin{array}{c}\text { Animal's } \\
\text { number }\end{array}$} & \multicolumn{4}{|c|}{ Sympathetic Nerve Activity } & & & \multicolumn{3}{|c|}{ Sarcomere Length $(\mu \mathrm{m})$} & & \multicolumn{2}{|c|}{ A:I ratio } \\
\hline & \multicolumn{3}{|c|}{ Frequency (rate/min) } & \multicolumn{2}{|c|}{ Amplitude $(\mathrm{mm})$} & & & & & & & \\
\hline & \multirow[t]{2}{*}{ Baseline } & \multicolumn{2}{|c|}{$\begin{array}{l}\text { Dopamine } \\
(\mu g / \mathrm{kg} / \mathrm{min})\end{array}$} & \multirow[t]{2}{*}{ Baseline } & \multicolumn{2}{|c|}{$\begin{array}{l}\text { Dopamine } \\
(\mu \mathrm{g} / \mathrm{kg} / \mathrm{min})\end{array}$} & \multirow{2}{*}{ Baseline } & \multicolumn{2}{|c|}{$\begin{array}{l}\text { Dopamine } \\
(\mu \mathrm{g} / \mathrm{kg} / \mathrm{min})\end{array}$} & \multirow{2}{*}{ Baseline } & \multicolumn{2}{|c|}{$\begin{array}{l}\text { Dopamine } \\
(\mu \mathrm{g} / \mathrm{kg} / \mathrm{min})\end{array}$} \\
\hline & & 1 & 12 & & 1 & 12 & & 1 & 12 & & 1 & 12 \\
\hline 1 & 36.00 & 33.00 & 33.00 & 22.00 & 13.00 & 20.00 & 2.10 & 2.70 & 1.80 & 1.90 & 2.20 & 1.50 \\
\hline 2 & 42.00 & 30.00 & 36.00 & 21.00 & 18.00 & 18.00 & 2.25 & 3.10 & 2.10 & 2.20 & 2.70 & 1.80 \\
\hline 3 & 45.00 & 36.00 & 39.00 & 20.00 & 15.00 & 17.00 & 2.30 & 3.10 & 1.90 & 1.64 & 2.66 & 1.30 \\
\hline 4 & 42.00 & 27.00 & 42.00 & 19.00 & 14.00 & 19.00 & 2.00 & 2.60 & 1.60 & 1.80 & 2.50 & 1.55 \\
\hline 5 & 36.00 & 30.00 & 39.00 & 21.00 & 12.00 & 20.00 & 2.50 & 3.20 & 1.50 & 2.40 & 3.00 & 2.00 \\
\hline 6 & 39.00 & 33.00 & 36.00 & 22.00 & 16.00 & 21.00 & 2.20 & 2.20 & 2.20 & 2.35 & 2.80 & 1.85 \\
\hline $\begin{array}{l}\text { Mean } \\
\pm \mathrm{SE}\end{array}$ & $\begin{array}{l}40.00 \\
\pm 1.48\end{array}$ & $\begin{array}{l}31.50 \\
\pm 1.28\end{array}$ & $\begin{array}{l}37.50 \\
\pm 1.28\end{array}$ & $\begin{array}{l}20.83 \\
\pm 0.48\end{array}$ & $\begin{array}{l}14.67 \\
\pm 0.88\end{array}$ & $\begin{array}{l}19.17 \\
\pm 0.60\end{array}$ & $\begin{array}{c}2.23 \\
\pm 0.07\end{array}$ & $\begin{array}{c}2.82 \\
\pm 0.16\end{array}$ & $\begin{array}{c}1.85 \\
\pm 0.11\end{array}$ & $\begin{array}{c}2.05 \\
\pm 0.13\end{array}$ & $\begin{array}{c}2.64 \\
\pm 0.11\end{array}$ & $\begin{array}{c}1.67 \\
\pm 0.11\end{array}$ \\
\hline$P$ value & & $P<0.001$ & $P>0.05$ & & $P<0.001$ & $P>0.05$ & & $P<0.01$ & $P<0.05$ & & $P<0.01$ & $P<0.05$ \\
\hline$\%$ Change & & $21.25 \downarrow$ & $6.25 \downarrow$ & & $29.75 \downarrow$ & $7.97 \downarrow$ & & $26.46 \uparrow$ & $\begin{array}{c}17.04 \\
\downarrow\end{array}$ & & $28.76 \uparrow$ & $18.54 \downarrow$ \\
\hline
\end{tabular}

SE: Standard Error.

$\downarrow$ : Percent decrease, $\uparrow:$ Percent increase.

Significance versus baseline level using one way ANOVA test 
Table (5): Effects of D1- and $\mathrm{D}_{2}$-like receptor activation on mean arterial blood pressure, heart rate, cardiac contractility and sympathetic nerve activity in adult male rabbits.

\begin{tabular}{|c|c|c|c|c|c|c|c|c|c|c|c|}
\hline & \multirow{3}{*}{ Variables } & \multicolumn{2}{|c|}{$\begin{array}{l}\text { Mean Blood Pressure } \\
\text { (mmHg) }\end{array}$} & \multicolumn{2}{|c|}{$\begin{array}{l}\text { Heart Rate } \\
\text { (beat/min) }\end{array}$} & \multicolumn{2}{|c|}{$\begin{array}{l}\text { Cardiac Contractility } \\
(\mathrm{mm})\end{array}$} & \multicolumn{3}{|c|}{ Sympathetic Nerve Activity } & \\
\hline & & \multirow[b]{2}{*}{ Baseline } & \multirow[b]{2}{*}{ Treatment } & \multirow[b]{2}{*}{ Baseline } & \multirow[b]{2}{*}{ Treatment } & \multirow[b]{2}{*}{ Baseline } & \multirow[b]{2}{*}{ Treatment } & \multicolumn{2}{|c|}{ Frequency (rate/min) } & \multicolumn{2}{|c|}{ Amplitude (mm) } \\
\hline & & & & & & & & Baseline & Treatment & Baseline & Treatment \\
\hline \multirow{4}{*}{ 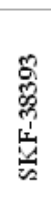 } & Mean & 86.33 & 7200 & 288.30 & 28500 & 34.17 & 3283 & 4000 & 3900 & 2083 & 1950 \\
\hline & $\pm \mathrm{SE}$ & \pm 2.80 & \pm 3.19 & \pm 6.54 & \pm 4.28 & \pm 2.68 & \pm 2.65 & \pm 1.48 & \pm 1.10 & \pm 0.48 & \pm 0.56 \\
\hline & $P$ value & & $P<0.01$ & & $P>0.05$ & & $P>0.05$ & & $P>0.05$ & & $P>0.05$ \\
\hline & $\%$ Change & & $16.60 \downarrow$ & & $1.14 \downarrow$ & & $3.92 \downarrow$ & & $2.50 \downarrow$ & & $6.39 \downarrow$ \\
\hline \multirow{4}{*}{ 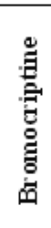 } & Mean & 89.75 & 75.33 & 291.70 & 280.00 & 33.50 & 24.50 & 40.00 & 33.00 & 20.83 & 15.00 \\
\hline & $\pm \mathrm{SE}$ & \pm 3.03 & \pm 3.46 & & \pm 6.83 & \pm 2.95 & \pm 3.81 & \pm 1.48 & \pm 1.10 & \pm 0.48 & \pm 0.89 \\
\hline & Significance & & $P<0.05$ & & $P>0.05$ & & $P<0.01$ & & $P<0.001$ & & $P<0.001$ \\
\hline & $\%$ Change & & $15.97 \downarrow$ & & $4.01 \downarrow$ & & $26.86 \downarrow$ & & $17.50 \downarrow$ & & $27.99 \downarrow$ \\
\hline
\end{tabular}

SE: Standard Error.

$\downarrow$ : Percent decrease

Significance versus baseline level using Paired two-sided Student "t-test". 
Table (6): Intravenous dopamine infusion (1and $12 \mu \mathrm{g} / \mathrm{kg} / \mathrm{min}$ ) after pre - treatment with $\mathrm{D}_{1}$-like (SCH-23390) and D2-like (metoclopramide) receptor blockers on mean arterial blood pressure, heart rate and cardiac contractility in adult male rabbits.

\begin{tabular}{|c|c|c|c|c|c|c|c|c|c|c|c|c|c|}
\hline & \multirow{3}{*}{ Variables } & \multicolumn{4}{|c|}{$\begin{array}{l}\text { Mean Blood Pressure } \\
\text { (mm Hg) }\end{array}$} & \multicolumn{4}{|c|}{$\begin{array}{l}\text { Heart Rate } \\
\text { (beat } / \mathrm{min} \text { ) }\end{array}$} & \multicolumn{4}{|c|}{$\begin{array}{c}\text { Cardiac Contractility } \\
(\mathrm{mm})\end{array}$} \\
\hline & & \multirow[t]{2}{*}{ Baseline } & \multirow{2}{*}{$\begin{array}{l}\text { Antagonist } \\
\text { (Control) }\end{array}$} & \multicolumn{2}{|c|}{$\begin{array}{l}\text { Dopamine } \\
(\mu g / \mathrm{kg} / \mathrm{min})\end{array}$} & \multirow[t]{2}{*}{ Baseline } & \multirow{2}{*}{$\begin{array}{l}\text { Antagonist } \\
\text { (Control) }\end{array}$} & \multicolumn{2}{|c|}{$\begin{array}{l}\text { Dopamine } \\
(\mu \mathrm{g} / \mathrm{kg} / \mathrm{min})\end{array}$} & \multirow[t]{2}{*}{ Baseline } & \multirow{2}{*}{$\begin{array}{l}\text { Antagonist } \\
\text { (Control) }\end{array}$} & \multicolumn{2}{|c|}{$\begin{array}{l}\text { Dopamine } \\
(\mu \mathrm{g} / \mathrm{kg} / \mathrm{min})\end{array}$} \\
\hline & & & & 1 & 12 & & & 1 & 12 & & & 1 & 12 \\
\hline \multirow{3}{*}{ 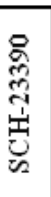 } & $\begin{array}{l}\text { Mean } \\
\pm \mathrm{SE}\end{array}$ & $\begin{array}{l}85.17 \\
\pm 2.37\end{array}$ & $\begin{array}{l}86.35 \\
\pm 2.41\end{array}$ & $\begin{array}{l}74.17 \\
\pm 3.58\end{array}$ & $\begin{array}{l}115.00 \\
\pm 3.39\end{array}$ & $\begin{array}{l}288.30 \\
\pm 6.54\end{array}$ & $\begin{array}{l}290.00 \\
\pm 5.16\end{array}$ & $\begin{array}{l}285.00 \\
\pm 4.28\end{array}$ & $\begin{array}{c}331.7 \\
\pm 11.38\end{array}$ & $\begin{array}{l}34.17 \\
\pm 2.68\end{array}$ & $\begin{array}{l}33.50 \\
\pm 2.31\end{array}$ & $\begin{array}{l}20.83 \\
\pm 1.08\end{array}$ & $\begin{array}{l}40.00 \\
\pm 2.85\end{array}$ \\
\hline & Significance & & $P>0.05$ & $\begin{array}{c}{ }^{{ }^{*} P} \\
<0.05\end{array}$ & $\begin{array}{l}{ }^{9} P \\
<0.01\end{array}$ & & $P>0.05$ & $\begin{aligned} & * P \\
&>0.05\end{aligned}$ & $\begin{array}{c}{ }^{*} P \\
<0.05\end{array}$ & & $P>0.05$ & ${ }^{*} P<0.01$ & ${ }^{*} P<0.01$ \\
\hline & \%Change & & $1.39 \uparrow$ & $14.12 \downarrow$ & $33.18 \uparrow$ & & $0.59 \uparrow$ & $1.72 \downarrow$ & $14.37 \uparrow$ & & $1.96 \downarrow$ & $39.01 \downarrow$ & $19.40 \uparrow$ \\
\hline \multirow{3}{*}{ 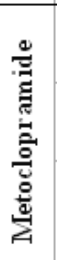 } & $\begin{array}{l}\text { Mean } \\
\pm \mathrm{SE}\end{array}$ & $\begin{array}{l}82.57 \\
\pm 2.88\end{array}$ & $\begin{array}{l}83.33 \\
\pm 3.65\end{array}$ & $\begin{array}{l}70.83 \\
\pm 3.93\end{array}$ & $\begin{array}{l}105.00 \\
\pm 2.19\end{array}$ & $\begin{array}{l}291.70 \\
\pm 3.07\end{array}$ & $\begin{array}{l}293.30 \\
\pm 2.11\end{array}$ & $\begin{array}{l}296.7 \\
\pm 4.94\end{array}$ & $\begin{array}{c}343.3 \\
\pm 14.53\end{array}$ & $\begin{array}{l}33.50 \\
\pm 2.95\end{array}$ & $\begin{array}{l}33.00 \\
\pm 2.71\end{array}$ & $\begin{array}{l}32.83 \\
\pm 2.61\end{array}$ & $\begin{array}{l}41.17 \\
\pm 1.33\end{array}$ \\
\hline & Significance & & $P>0.05$ & $\begin{array}{c}{ }^{*} P \\
<0.05\end{array}$ & $\begin{array}{l}{ }^{*} P \\
<0.01\end{array}$ & & $P>0.05$ & $\begin{array}{l}* P \\
>0.05\end{array}$ & $\begin{array}{l}{ }^{*} P \\
<0.01\end{array}$ & & $P>0.05$ & $* P>0.05$ & ${ }^{*} P<0.01$ \\
\hline & $\%$ Change & & $0.92 \uparrow$ & $14.92 \downarrow$ & $26.00 \uparrow$ & & $0.55 \uparrow$ & $1.16 \uparrow$ & $17.05 \uparrow$ & & $0.15 \downarrow$ & $0.52 \downarrow$ & $24.70 \uparrow$ \\
\hline
\end{tabular}

SE : Standard Error.

$\downarrow$ : Percent decrease, $\uparrow:$ Percent increase.

P: Significance versus baseline level using Paired two-sided Student "t-test"; *P significance versus control using one way ANOVA test. 
Table (7): Effect of intravenous dopamine infusion (1 and $12 \mu \mathrm{g} / \mathrm{kg} / \mathrm{min}$ ) after pre-treatment with $\alpha$-adrenergic receptor blocker (reserpine) and $\beta$-adrenergic receptor blocker (propranolol) on mean arterial blood pressure, heart rate and cardiac contractility in adult male rabbits.

\begin{tabular}{|c|c|c|c|c|c|c|c|c|c|c|}
\hline & \multirow{3}{*}{ Variables } & \multicolumn{3}{|c|}{$\begin{array}{c}\text { Mean Blood Pressure } \\
(\mathrm{mm} \mathrm{Hg})\end{array}$} & \multicolumn{3}{|c|}{$\begin{array}{l}\text { Heart Rate } \\
\text { (beat } / \mathrm{min} \text { ) }\end{array}$} & \multicolumn{3}{|c|}{$\begin{array}{l}\text { Cardiac Contractility } \\
(\mathrm{mm})\end{array}$} \\
\hline & & \multirow[t]{2}{*}{ Control } & \multicolumn{2}{|c|}{$\begin{array}{l}\text { Dopamine } \\
(\mu \mathrm{g} / \mathrm{kg} / \mathrm{min})\end{array}$} & \multirow[t]{2}{*}{ Control } & \multicolumn{2}{|c|}{$\begin{array}{l}\text { Dopamine } \\
(\mu \mathrm{g} / \mathrm{kg} / \mathrm{min})\end{array}$} & \multirow[t]{2}{*}{ Control } & \multicolumn{2}{|c|}{$\begin{array}{l}\text { Dopamine } \\
(\mu \mathrm{g} / \mathrm{kg} / \mathrm{min})\end{array}$} \\
\hline & & & 1 & 12 & & 1 & 12 & & 1 & 12 \\
\hline \multirow{3}{*}{ 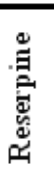 } & Mean \pm SE & $\begin{array}{l}83.12 \pm \\
3.15\end{array}$ & $71.11 \pm 3.09$ & $82.42 \pm 2.52$ & $\begin{array}{c}288.30 \pm 7.9 \\
2\end{array}$ & $\begin{array}{c}291.70 \pm 7.9 \\
2\end{array}$ & $\begin{array}{c}330.00 \pm 7.3 \\
0\end{array}$ & $38.17 \pm 1.70$ & $27.50 \pm 1.67$ & $44.33 \pm 2.04$ \\
\hline & Significance & & $P<0.05$ & $P>0.05$ & & $P>0.05$ & $P<0.05$ & & $P<0.01$ & $P<0.01$ \\
\hline & $\%$ Change & & $14.45 \downarrow$ & $0.84 \downarrow$ & & $1.18 \uparrow$ & $14.45 \uparrow$ & & $35.24 \downarrow$ & $16.14 \uparrow$ \\
\hline \multirow{3}{*}{ 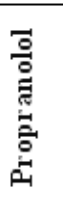 } & Mean \pm SE & $82.67 \pm 2.31$ & $69.00 \pm 2.39$ & $94.52 \pm 3.96$ & $\begin{array}{c}260.00 \pm 5.7 \\
7\end{array}$ & $\begin{array}{c}256.70 \pm 7.6 \\
0\end{array}$ & $\begin{array}{c}256.70 \pm 5.5 \\
8\end{array}$ & $36.33 \pm 1.28$ & $26.67 \pm 2.43$ & $33.83 \pm 0.60$ \\
\hline & Significance & & $P<0.01$ & $P<0.05$ & & $P>0.05$ & $P>0.05$ & & $P<0.01$ & $P>0.05$ \\
\hline & $\%$ Change & & $16.54 \downarrow$ & $14.33 \uparrow$ & & $1.27 \downarrow$ & $1.27 \downarrow$ & & $26.62 \downarrow$ & $6.88 \downarrow$ \\
\hline
\end{tabular}

SE: Standard Error.

Significance versus control using one way ANOVA test.

$\downarrow$ : Percent decrease, $\uparrow$ : Percent increase. 


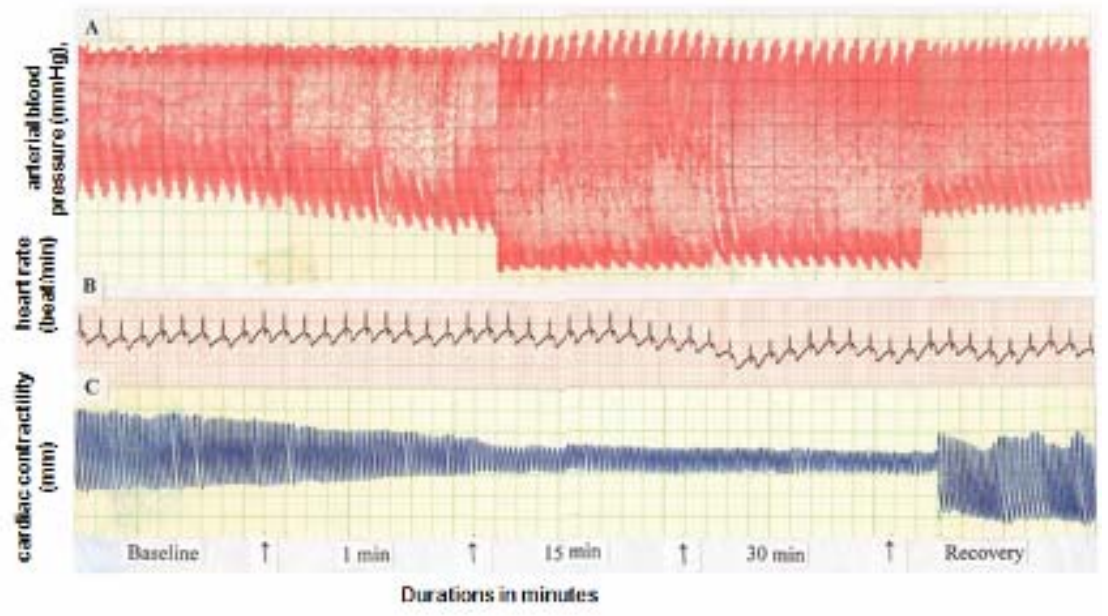

Figure (1): Recording of arterial blood pressure (A), heart rate (B) and cardiac contractility (C) responses to intravenous dopamine infusion at a rate of $1 \mu \mathrm{g} / \mathrm{kg} / \mathrm{min}$ in adult male rabbits.

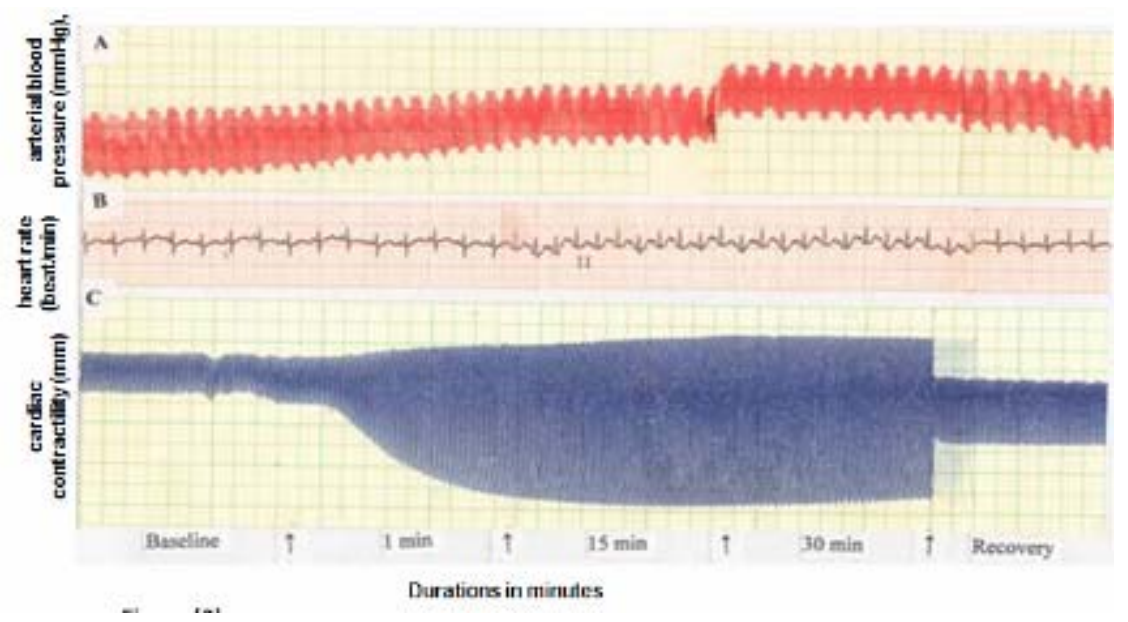

Figure (2): Recording of arterial blood pressure (A), heart rate (B) and cardiac contractility (C) responses to intravenous dopamine infusion at a rate of $12 \mu \mathrm{g} / \mathrm{kg} / \mathrm{min}$ in adult male rabbits. 


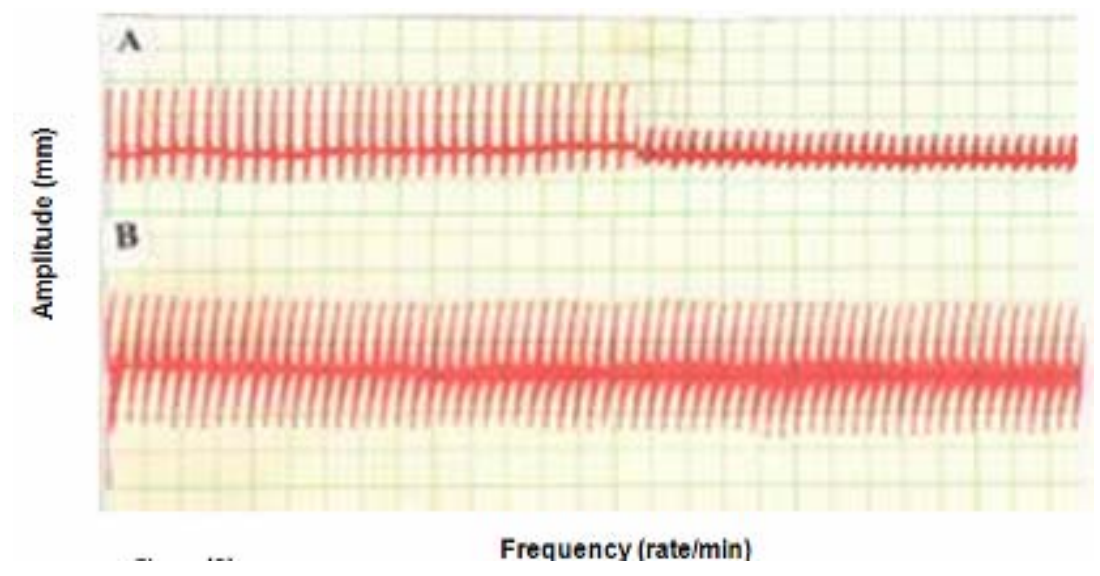

Figure (3): Recording of sympathetic nerve activity response to intravenous dopamine infusion at a rate of $1 \mu \mathrm{g} / \mathrm{kg} / \mathrm{min}$ (A) and $12 \mu \mathrm{g} / \mathrm{kg} / \mathrm{min}$ (B) in adult male rabbits.

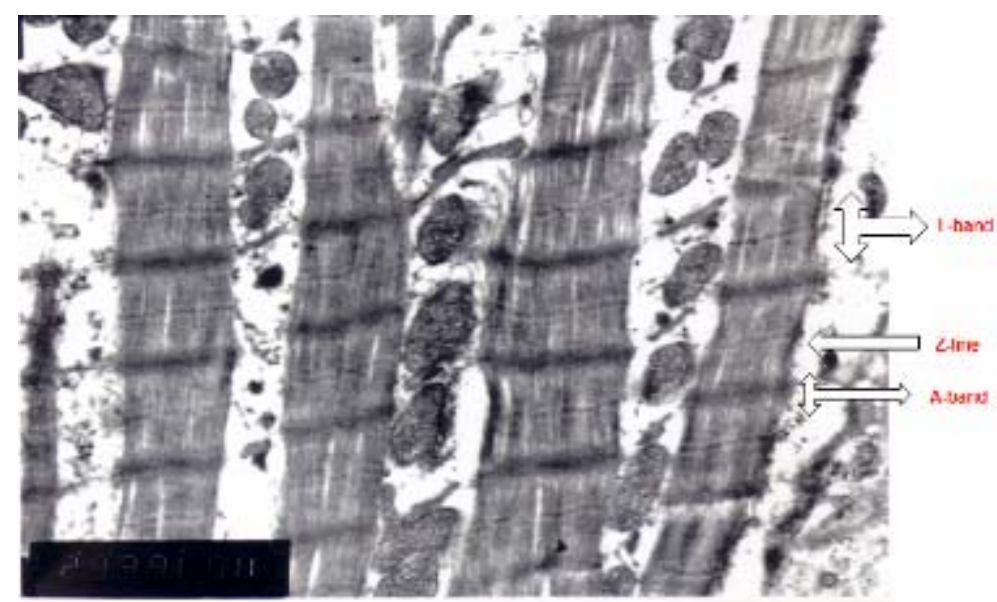

Fig (4): Electron micrograph of longitudinal section of cardiac muscle of control rabbits shows the $\mathrm{Z}$ disc, I, $\mathrm{A}$ and $\mathrm{H}$ bands and $\mathrm{M}$ line are readily defined. $(\mathrm{X} 8,000)$ 


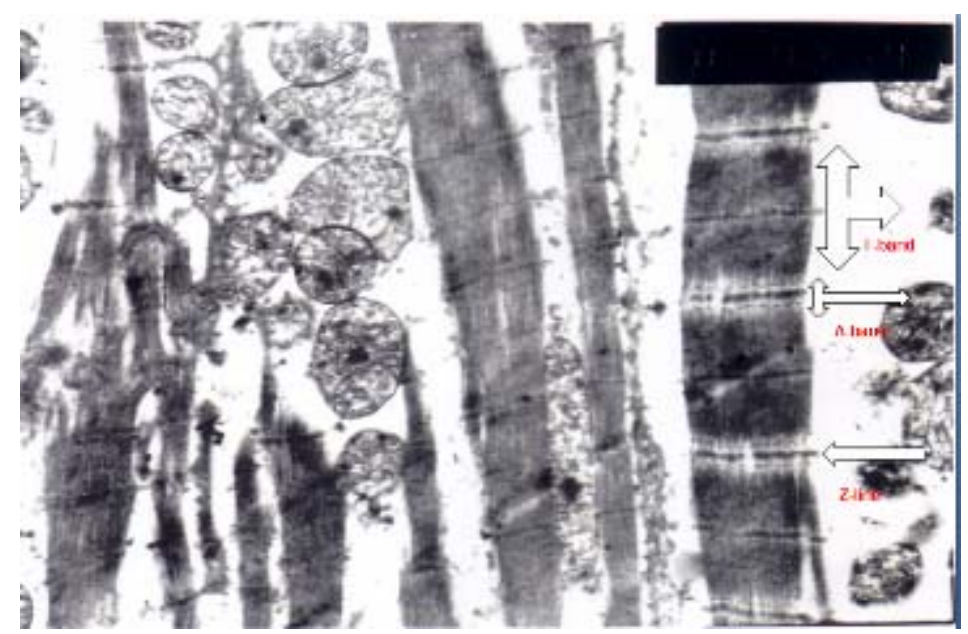

Fig (5): Electron micrograph of longitudinal section of rabbit's cardiac muscle after intravenous dopamine infusion at a rate of $1 \mu \mathrm{g} / \mathrm{kg} / \mathrm{min}$, shows the relaxation of myofibrils (as evident by A: I ratio) $(X 8,000)$

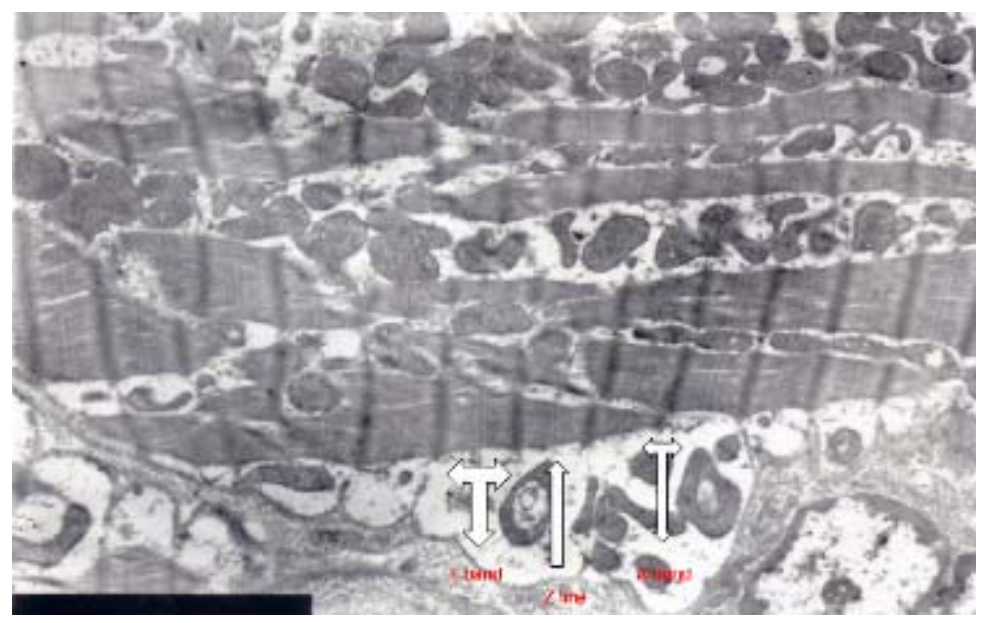

Fig (6): Electron micrograph of longitudinal section of rabbit's cardiac muscle after intravenous dopamine infusion at a rate of $12 \mu \mathrm{g} / \mathrm{kg} / \mathrm{min}$, shows the contraction of myofibrils (as evident by A: I ratio) $(\mathrm{X} 8,000)$ 


\section{DISCUSSION}

The pharmacological effects of dopamine are unique sequence of receptor activation and dose dependent. The observations of the present study demonstrated that dopamine elicits biphasic effect on $\mathrm{ABP}$ and CC. At low infusion rates of dopamine $(1 \mu \mathrm{g} / \mathrm{kg} / \mathrm{min})$, MBP and $\mathrm{CC}$ were decreased, whereas at high infusion rates $(12 \mu \mathrm{g} / \mathrm{kg} / \mathrm{min}), \mathrm{MBP}$, $\mathrm{HR}$ and $\mathrm{CC}$ were increased. The effect of dopamine infusion was apparent immediately and gradually (by $1 \mathrm{~min}$ ), maximal change was seen during the first $15 \mathrm{~min}$ of infusion and tended to return to the baseline levels by the end of infusion. Thus the effects of dopamine occur only during the infusion periods. With dose of $1 \mu \mathrm{g} / \mathrm{kg} / \mathrm{min}$ of dopamine infusion, the depressor effect resulted mainly from decrease in the diastolic blood pressure, whereas the pressor effect seen with a dose of $12 \mu \mathrm{g} / \mathrm{kg} / \mathrm{min}$ caused by elevations of both diastolic and systolic blood pressure.

A decrease in MBP in the absence of changes in HR as observed after dopamine at low infusion rate $(1 \mu \mathrm{g} / \mathrm{kg} / \mathrm{min})$ in the present study suggests a vasodilatation. The cardiovascular effects of exogenously administered dopamine in different animals and human were investigated sparsely. Dobrowolski et al. ${ }^{(\mathbf{1 8})}$ reported that infusion of dopamine caused vasodilatation in rabbits. Olsen $^{(19)}$ found that with 1 and 2 $\mu \mathrm{g} / \mathrm{kg} / \mathrm{min}$ of dopamine the MBP was decreased in human. Low dose dopamine $(2-5 \mu \mathrm{g} / \mathrm{kg} / \mathrm{min})$ has been used to augment renal perfusion in critically ill surgical patients ${ }^{(\mathbf{2 0})}$. Schenarts et al. ${ }^{(21)}$ reported that low dose dopamine increase renal blood flow.

Vascular actions of dopamine are mediated primarily through its interaction with specific receptors located in the vascular system. However, only limited information is available concerning the molecular nature and subtype(s) of cardiovascular dopamine receptors in rabbits, so their functions are not established yet. In rabbits, dopamine receptors are located in the renal, mesenteric, pulmonary, coronary and splenic arteries ${ }^{(\mathbf{2 2 , 2 3 , 2 4 , 2 5})}$. In this study, $\mathrm{D}_{1}$-like receptor activation with $\mathrm{SKF}$ $38393(5 \mu \mathrm{g} / \mathrm{kg} / \mathrm{min})$ and $\mathrm{D}_{2}$-like receptor activation with bromocriptine $(1 \mu \mathrm{g} / \mathrm{kg} / \mathrm{min}) \quad$ decreased MBP compared with baseline levels. This observation is similar to the hypotensive effect of other $D_{1}$-like agonists such as fenoldopam and apomorphine (26) and $\mathrm{D}_{2}$-like receptors agonists, such as pengolide and quipinole ${ }^{(26)}$. For confirmation of the suggestion that dopamine acts via dopaminergic receptors at low infusion rates, dopamine $(1 \mu \mathrm{g} / \mathrm{kg} / \mathrm{min})$ was iv infused after pre-treatment with selective $\mathrm{D}_{1}$-like antagonist SCH-23390 (50 $\mu \mathrm{g} / \mathrm{kg}$, ip) and $\mathrm{D}_{2}$-like antagonist metoclopramide (15 $\mathrm{mg} / \mathrm{kg}$, ip). $\mathrm{D}_{1^{-}}$and $\mathrm{D}_{2}$-like receptor blockade attenuated but didn't abolish the hypotensive response to dopamine. Dopamine interaction with adrenergic receptors was, also, investigated in this work. Dopamine infusion $(1 \mu \mathrm{g} / \mathrm{kg} / \mathrm{min})$ into animals pre-treated with reserpine $(\alpha$ adrenergic receptor blocker) and 
propranolol ( $\beta$-adrenergic receptor blocker) caused decrease in MBP. Chemical sympathectomy didn't modify the extent of inhibition of MBP by dopamine.

In this study, activation of $\mathrm{D}_{2}$-like receptors diminished not only MBP but also, RSNA. These results suggest that dopamine, via $D_{1}$-and $D_{2}$-like receptors, has a hypotensive effect at low infusion rates. Stimulation of $\mathrm{D}_{1^{-}}$ like receptors was suggested to cause direct hypotensive effects whereas, stimulation of $\mathrm{D}_{2}$-like receptors was suggested to inhibit norepinephrine release to indirectly induce hypotension. Biochemical and pharmacological evidence has suggested a postjunctional localization of $\mathrm{D}_{1}$-like receptors and their stimulation causes direct vasorelaxation and reduction of vascular resistance. Although, investigation of the mechanism of $\mathrm{D}_{1}$ like receptors activation was not an objective of this study, there are several potential explanations. The vasodilator effect of dopamine via $\mathrm{D}_{1}$ like receptors is mediated mainly by cAMP/ protein kinase A (PKA) ${ }^{(27)}$. Protein kinase A stimulates a phosphatase enzyme which, in turn, dephosphorylates calcium channels leading to their inactivation and inhibition of inward calcium currents $^{(28)}$. There are experimental reports that dopamine depresses the cardiac functions through the inhibition of catecholamine release via stimulation of D2-receptors in addition to postsynaptic $\mathrm{D}_{1}$-receptor mediated vasodilatation. Yoon et al. ${ }^{(29)}$ hypothesized that the increased norepinepherine concentration in the synaptic cleft might be required for elicitation of cardiovascular depression through dopaminergic receptors. $\mathrm{D}_{2}$-like receptors were considered mainly prejunctional and their stimulation induces indirect vasorelaxation by modulating

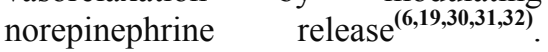
Accordingly, stimulation of postjunctional $\quad\left(\mathrm{D}_{1}\right.$-like $) \quad$ or prejunctional $\left(\mathrm{D}_{2}\right.$-like $)$ dopamine receptors may represent a therapeutic principle in hypertension treatment.

Dopamine at low dose $(1 \mu \mathrm{g} / \mathrm{kg} / \mathrm{min})$, apart from its action on $\mathrm{ABP}$, has been observed to cause decreased $\mathrm{CC}$. In the same time, sarcomere length and A:I ratio were increased after $1 \mu \mathrm{g} / \mathrm{kg} / \mathrm{min}$ dopamine treatment which will contribute to a decrease in developed force ${ }^{(33)}$. In addition, $\mathrm{CC}$ decreased not only after $\mathrm{D}_{2}$-like receptor activation but also after $\mathrm{D}_{1}$-like receptor blockade. Cardiac contractility, also, decreased after $\beta$-adrenergic receptor blockade. According to these data, dopamineinduced cardiac relaxation at low doses suggests to be mediated by dopamine receptors and is $\mathrm{D}_{2}$-like dependant by modulating norepinephrine release. Activation of presynaptic $D_{2}$ receptors inhibits neuronal norepinepherine release from renal sympathetic nerve endings ${ }^{(34)}$. However, only limited information is available concerning the profile and the subtypes in the heart. The presence of $D_{1}$ and $D_{2}$ receptors has been reported in rat and human cardiac tissue ${ }^{(35,36)}$. $\mathrm{D}_{2}$-like receptors in the heart were mainly postjunctional and located primarily in atrial tissue ${ }^{(37)}$. Gomez et al. ${ }^{(38)}$ and Ricci et al. ${ }^{(39)}$ by autoradiographic studies of guinea pig hearts support 
the presence of $D_{3}$ and $D_{4}$ receptors in the right and left ventricle. Yoon et al. (29) hypothesized that the increased norepinepherine concentration in the synaptic cleft might be required for elicitation of cardiovascular depression through dopaminergic receptors. Ozono et al. $^{(35)}$ demonstrated postjunctional $\mathrm{D}_{1^{-}}$ receptors on myocardial sarcolemma of rats, the stimulation of which caused a small but significant increase in cAMP concentration. Several previous experimental studies also indicated no significant role of $D_{1}$ receptors in the inotropic effects of dopamine on isolated guinea-pig $\operatorname{atria}^{(40)}$.

In contrast to a decrease of MBP and $\mathrm{CC}$ after low infused doses of dopamine $(1 \mu \mathrm{g} / \mathrm{kg} / \mathrm{min})$, an increase in MBP, HR and CC was observed after high infusion doses of dopamine (12 $\mu \mathrm{g} / \mathrm{kg} / \mathrm{min}$ ). To clarify whether these hypertensive effects of dopamine as well as the chronotropic and inotropic ones are direct actions on the cardiac muscles or indirect ones by releasing noradrenaline from the sympathetic nerve terminals in the cardiac muscles, first the dopamine receptor subtype(s) involved in the cardiovascular effect of dopamine at high doses was investigated. This study demonstrated that iv infusion of dopamine at a rate of $12 \mu \mathrm{g} / \mathrm{kg} / \mathrm{min}$ after pre-treatment with $\mathrm{D}_{1}$-like antagonist (SCH-23390) and $\mathrm{D}_{2}$-like antagonist (metoclopramide) increased MBP, HR and CC. Thus, $\mathrm{D}_{1}$ and $\mathrm{D}_{2}$-like receptors are not important in the vascular and cardiac effects of the higher doses of dopamine. In an attempt to investigate the interaction between dopamine and sympathetic nervous system, 12 $\mu \mathrm{g} / \mathrm{kg} / \mathrm{min}$ dopamine infusion resulted in an increase in RSNA. In addition, a chemical denervation of the sympathetic nerve terminals was made by a pre-treatment of the animals with reserpine and propranolol. This experiment revealed that dopamine infusion at high rate (12 $\mu \mathrm{g} / \mathrm{kg} / \mathrm{min}$ ) into animals after reserpine caused increase in HR and $\mathrm{CC}$ and after propranolol caused increase in MBP when compared with pre-injected dopamine level (control level). These results collectively suggest that vascular and cardiac actions of higher dose of dopamine are mediated by the activation of $\alpha$ and $\beta$-adrenoceptors. The arterial blood pressure effect is $\alpha$-adrenergic dependant, whereas HR and $\mathrm{CC}$ was $\beta$-adrenergic dependant. These results are in agreement with Olsen (19) who reported that with increasing doses of dopamine (at and above 7.5 $\mu \mathrm{g} / \mathrm{kg} / \mathrm{min}$ ), the renal blood flow was decreasing. Furukawa et al. ${ }^{(41)}$ found that in dogs, improvement in hemodynamics indicated by marked increase in cardiac blood flow, cardiac output and mean arterial blood pressure was observed at higher doses of dopamine (10 and $20 \mu \mathrm{g} / \mathrm{kg} / \mathrm{min}$ ). Meneton $^{(\mathbf{4 2})}$ stated that at higher concentrations of dopamine, $\alpha$ - and $\beta$ adrenergic receptors are occupied. Jasiñska et al. ${ }^{(43)}$ found that dopamine infusion at $10 \mu \mathrm{g} / \mathrm{min} / \mathrm{kg}$ improved myocardial contractility in rabbits. Recently, Wakita $^{(44)}$ observed that administration of dopamine into the perfusate of the isolated rabbit heart at doses of 100 to $1000 \mu \mathrm{g}$ was found to produce dose-related increases in contractile activities and heart rates in 
the normal preparation, and this effect was greatly suppressed in the denervated preparations, suggesting that the primary chronotropic and inotropic effect of dopamine is an indirect one via norepinephrine release of from sympathetic nerve terminals.

It is evident from the forgoing discussion on multiple dopamine receptors that activation and /or blockade of these receptors can lead to pronounced changes in cardiovascular functions. Therefore, an obvious question that can be asked is whether sufficient quantities of endogenous dopamine are available to act on these receptors and thereby play a physiological role in control of cardiovascular function. In the present study, $\mathrm{D}_{1}$-like receptor antagonist SCH-23390 and $\mathrm{D}_{2}$-like receptor antagonist metoclopramide were used to investigate the interaction between endogenous dopamine and ABP, HR and $\mathrm{CC}$ in normal rabbits. Blockade of either $\mathrm{D}_{1}$-like receptor by $\mathrm{SCH}-23390$ or $\mathrm{D}_{2}$-like receptors by metoclopramide alone has no effect on baseline levels of MBP, HR and CC. Furthermore, iv dopamine infusion alone at low rate (1 $\mu \mathrm{g} / \mathrm{kg} / \mathrm{min}$ ) decreased significantly MBP and CC, whereas change in HR wasn't significantly altered. These data suggest that under basal conditions endogenous, dopamine may not alter the basal cardiac and vascular functions. There is no physiological activation of cardiovascular dopaminergic receptors in rabbits. Oliva et al. ${ }^{(45)}$ claimed that microinjection of dopamine into the caudal nucleus tractus solitarii does not affect either the autonomic activity to the cardiovascular system or the autonomic and respiratory responses of chemoreflex activation in awaken rats.

There are two possible sources of endogenous dopamine which can activate these receptors. Circulating dopamine and dopamine containing neurons in close proximity to these receptors. Levels of circulating dopamine have been measured accurately in various animals and humans. The levels of free dopamine in plasma are too low to exert any significant effect. The affinity of dopamine to its receptors is in the nanomolar range. Circulating concentrations of dopamine (picomolar range) are not sufficiently high to activate dopamine receptors, while in dopamine producing tissues concentrations in the high nanomolar to micromolar range can be attained ${ }^{(4)}$. Circulating dopamine reportedly synthesized in neural and non neural tissues $^{(4)}$.

In conclusion. The results of these experiments indicate that dopamine elicit a biphasic effect on cardiovascular system in normal anesthetized adult male Balady rabbits. At low infusion rate of dopamine (1 $\quad \mu \mathrm{g} / \mathrm{kg} / \mathrm{min}) \quad$ a hypotensive and negative inotropic effects were observed, whereas at high infusion rates (4 \& 12 $\mu \mathrm{g} / \mathrm{kg} / \mathrm{min}$ ) dopamine has hypertensive, positive chronotropic and inotropic effects. The effect of dopamine infusion was apparent immediately (1 min) and gradually with maximal changes seen at $15 \mathrm{~min}$. At low infusion rate $(1 \mu \mathrm{g} / \mathrm{kg} / \mathrm{min})$, cardiovascular effect of dopamine 
predominantly due to stimulation of dopaminergic receptors $\left(\mathrm{D}_{1}\right.$-and $\mathrm{D}_{2}$ like receptors), but at high infusion rate $(12 \mu \mathrm{g} / \mathrm{kg} / \mathrm{min})$, action occurs via stimulation of $\alpha$ - and $\beta$-adrenergic receptors. The arterial blood pressure effect is $\alpha$-adrenergic dependent, whereas $\mathrm{HR}$ and $\mathrm{CC}$ were $\beta$ adrenergic dependent. These results demonstrated also, that $\mathrm{D}_{1^{-}}$and $\mathrm{D}_{2}$ like activation decreased MBP and CC. In the present study, at low rate of infusion, MBP, HR and CC were not significantly changed after $\mathrm{D}_{1-}$ and $\mathrm{D}_{2}$-like receptor blockade, whereas a significant decrease was observed after $\mathrm{D}_{1^{-}}$and $\mathrm{D}_{2}$ like activation. These data demonstrate that normal rabbit's endogenous dopamine may not alter the basal cardiovascular functions.

\section{Acknowledgement:}

The authors thank Dr Amal T Abou El- Ghait, Assistant professor of histology for making microscopic examinations of left venrticle.

\section{REFERENCES}

1. Jose PA, Raymond JR, Bates MD, Aperia A, Felder RA, Carey RM (1992). The renal dopamine receptors. J Am Soc Nephrol; 2(8):1265-1278.

2. Jose P, Gilbert M, Robin E, Felder C (2003). Regulation of blood pressure by dopamine receptors. Nephron Physiol; 95:19-27.

3. Gingrich J, Caron M (1993). Recent advances in the molecular biology of dopamine receptors. Annu Rev Neurosci; 16:299-321.

4. Jose PA, Eisner GM, Felder RA (1998). Renal dopamine receptors in health and hypertension. Pharmacol Ther; 80:149-182.

5. Amenta F, Ricci A, Rossodivita I, Avola R, Tayebati SK (2001). The dopaminergic system in hypertension. Clin Exp Hypertens; 23(1-2):15-24.

6. Amenta F, Ricci A, Tayebati SK \& Zaccheo D (2002). The peripheral dopaminergic system: morphological analysis, functional and clinical applications. J Anat Embryol; 107(3):145-167.

7. Jose PA, Eisner GM, Felder RA (2002). Role of dopamine receptors in the kidney in the regulation of blood pressure. Current Opinion in Nephrology and Hypertension; 11:87-92.

8. Bek M, Zheng S, Asico L, Grandy D, Rubinstein M, Carey R, Eisner G, Jose PA (2001). P-547: $\quad \mathrm{D}_{4}$ dopamine receptor regulates blood pressure via V1 and AT1 receptors. Am J Hypertens; 14:213A-214A.

9. Muhlbauer B, Gleiter C, Gies C, Luippold G, Loschmann P (1997). Renal response to infusion of dopamine precursors in anasthetized rats. Naunyn Schmiedebergs Arch Pharmacol; 356:838-845.

10. Gue M, Gleizes-Escala C, RioLacheze $\mathbf{C}$, Junien $\mathbf{J}$ and Bueno L (1994). Reversal of CRF- and dopamine induced stimulation of colonic motility by $\mathrm{CCK}$ and igmesine (Jo 1784) in the rat. Br J Pharmacol; 111:930-934.

11. Bazzani $C$, Nardi $M$, Ferrante F, Bertolini A, Guarini S (1994).

Dopamine $\mathrm{D}_{1}$ receptors are 
involved in the ACTH-induced reversal of hemorrhagic shock. Eur J Pharmacol; 253(3) : 303306.

12. Kaya $F$, van Duin CT, VanMiert AS (1994). Effects of dopamine receptor agonists on food intake and rumen motility in dwarf goats. J Vet Pharmacol Ther; 17(2):120-126.

13. Slavik KJ, LaPointe J (1993). Involvement of inhibitory dopamine-2 receptors in resting bradycardia in exerciseconditioned rats. J Appl Physiol; 74(5):2086-2091.

14. Okada K, Shinozuka K, Shimoura K, Kobayashi Y, Hattori K, Nakase A (1993). Effects of reserpine on the content and uptake of dopamine and noradrenaline in rabbit arteries. Clin Exp Pharmacol Physiol; 20(4):261-267.

15. Dhasmana $K$, Villalon $C$, Zhu Y, Parmar S (1993). The role of dopamine $\left(\mathrm{D}_{2}\right)$, alpha and betaadrenoceptor receptors in the decrease in gastrointestinal transit induced by dopamine and dopamine-related drugs in the rat. Pharmacol Res; 27(4):335-347.

16. Guyton AC, Hall JE. Textbook of medical physiology (2006), $11^{\text {th }}$ edition; pp175-177. Elsevier Inc., Philadelphia, Pennsylvania.

17. Thorén P, Ricksten SE (1979). Recordings of renal and splanchnic sympathetic nervous activity in normotensive and spontaneously hypertensive rats. Clin Sci (Lond); 57(5):197s-199s.

18. Dobrowolski $\mathrm{K}$, Endlich $\mathbf{N}$, Sadowsk J, Steinhausen J (1997). Cardiovascular and renal effects of endothelin receptor blockade with PD145065 and interaction with urodilatin. Acta Physiol Scand; 159:7-13.

19. Olsen N (1998). Effects of dopamine on renal haemodynamics tubular function and sodium excretion in normal humans. Dan Med Bull; 45(3):282-297.

20. Eachempati S, Reed R (2003). Use of creatinine clearances to monitor the effect of low-dose dopamine in critically ill surgical patients. J Surg Res; 112(1) : 4348.

21. Schenarts PJ, Sagraves SG, Bard MR, Toschlog EA, Goettler CE, Newell MA, Rotondo MF (2006). Low-dose dopamine: a physiologically based review. Curr Surg; 63(3):219-225.

22. Munch G, Raether A, Schoffel E, Illes $P$ (1991). Postsynaptic dopamine $\mathrm{D}_{\mathrm{A} 1}$-and $\mathrm{D}_{\mathrm{A} 2}$-receptors in jejunal arteries of rabbits. J Cardiovasc 18(3):468-471.

23. Wang ZQ, Way $D$, Trigg $L$, McGrath BP (1992). Receptors mediate renal effects of dopamine prodrug, gludopa, in conscious rabbits. Clin Exp Pharmacol Physiol; 19(5):369-372.

24. Kobayashi Y, Ricci A, Amenta F, Cavallotti C, Hattori K (1995). Localization of dopamine receptors in the rabbit lung vasculature. J Vasc Res; 32:200206.

25. Bruzzone $P, D^{\prime}$ Andrea $V$, Motta C, Cavallotti C (2002). Occurrence of Dopaminergic $\left(\mathrm{D}_{2}\right)$ Receptors within the Rabbit 
Pulmonary Circulation.

Pulmonary Pharmacology \& Therapeutics; 15:393-398.

26. Velasco $M$, Luchsinger A (1998). Dopamine: Pharmacologic and therapeutic aspects. Am J Ther; 5(1):37-43.

27. Gao Y, Nishimura Y, Suzuki A (1995). Dopamine induced relaxation in isolated intrarenal arteries from adult stroke-prone spontaneously hypertensive rats. Clin Exp Pharmacol Physiol; 22(1):S96-S98.

28. Gardner B, Liu Z, Jiang D, Sibley D (2001). The role of phosphorylation/

dephosphorylation in agonistinduced desensitization of D1 dopamine receptor function: evidence for a novel pathway for receptor dephosphorylation. Mol Pharmacol; 59(2):310-321.

29. Yoon J, Ko C, Ahn Y, Park K, Choe K, Yoo K, Kim K, Kim S, Cho B (1994). Mechanism of decrease in heart rate by peripheral dopaminergic D2receptors. Yonsei Med J; 35(4):411-419.

30. Jose PA, Eisner GM, Felder RA (1999). Role of dopamine in pathogenesis of hypertension. Clin Exp Pharmacol Physiol; 26:S10-13.

31. Amenta F, Barili P, Bronzetti E, Felici L, Mignini F, Ricci A (2000). Localization of dopamine receptor subtypes in systemic arteries. Clin and Exper Hypertension; 22(3):277-288.

32. Tabbutt S, Helfaer M, Nichols D (2006). Pharmacology of cardiovascular drugs. In: Critical Heart Disease in Infants and
Children (ed. Nichols D, Ungerleider R, Spevak P, Greeley W, Cameron D, Lappe D, Wetzel R); pp. 173-203. Mosby Elsevier, Philadelphia.

33. Cecchi G, Bagni M (1994). Myofilament lattice spacing affects tension in striated muscle. News Physiol Sci; 9:3-7.

34. Rump L, Ruff G, Wolk V, Schollmeyer P (1991). Alpha 2adrenoceptor activation inhibits noradrenaline release in human and rabbit isolated renal arteries. Eur J Pharmacol; 196(3):277-283.

35. Ozono R, O'Connell D, Vaughan C, Botkin S, Walk S, Felder R, Carey R (1996). Expression of the subtype 1A dopamine receptor in the rat heart. Hypertension; 27:693-703.

36. Ricci A, Bronzetti E, Fedele F, Ferrante F, Zaccheo D, Amenta F (1998). Pharmacological characterization and autoradiographic localization of a putative dopamine D4 receptor in the heart. J Auton Pharmacol; 18:115-121.

37. Amenta F, Gallo P, Rossodivita A, Ricci A (1993). Radioligand binding and autoradiographic analysis of dopamine receptors in the human heart. NaunynSchmiedeberg's Arch Pharmacol; 347:147-154.

38. Gomez M, Rousseau G, Nadeau R, Berra R, Flores G, Suárez J (2002). Functional and autoradiographic characterization of dopamine D2-like receptors in the guinea pig heart. Can J Physiol Pharmacol; 80:578-587.

39. Ricci A, Marchal-Victorion S, Bronzetti E, Parini A, Amenta 
F, Tayebati S (2002). Dopamine D4 receptor expression in rat kidney: evidence for pre and post junctional localization. J Histochem Cytochem; 50:10911096.

40. Martinez-Mir I, Morales-Olivas F, Rubio E (1987). The lack of the effect of DA-1 and DA-2 dopamine agonists on the isolated guinea-pig atria. J Autonom Pharmacol; 7:111- 117.

41. Furukawa S, Nagashima $Y$, Hosi K, Hirao H, Tanaka R, Yamane $M$ (2002). Effects of dopamine infusion on cardiac and renal blood flows in dogs. $\mathrm{J}$ Vet Med Sci; 64(1):41-44.

42. Meneton $\mathbf{P}$ (2000). Comparative roles of the renal apical sodium transport systems in blood pressure control. J Am Soc Nephrol; 11(16):135-139.
43. Jasińska M, Owczarek J, Orszulak-Michalak D (2006). Influence of simvastatin at high dose and nifedipine on hemodynamic parameters in rabbits. Pharmacol Rep; 58(1):4859.

44. Wakita Y (2007). Inotropic, Chronotropic, and Arrhythmogenic Effects of Dopamine on the Isolated Working Heart of Rabbit. J Physiol Sci; 57(3):147-157.

45. Oliva WM, Granjeiro EM, Bongamba LG, Mendes RA, Machado BH (2010). Dopamine microinjected into brainstem of awake rats affects baseline arterial pressure but not chemoreflex responses. Auton Neurosci; 155(1-2):73-81. 


\title{
وظائف الجهاز الاورى والدوبامين: الية العمل فى الإئ

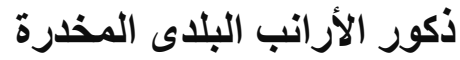

\author{
ابناس أحدد حامد؛ امتثال محمود مصطفى؛ نشوى على عبل المطلب المبا.

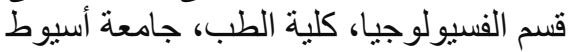

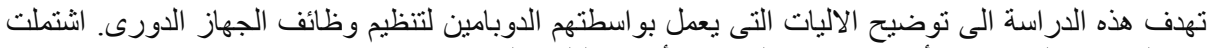

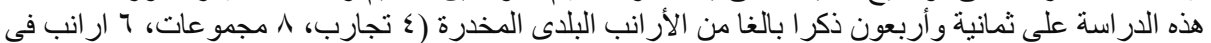

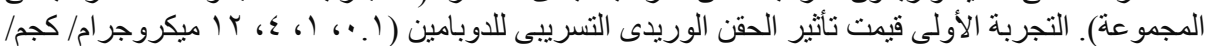

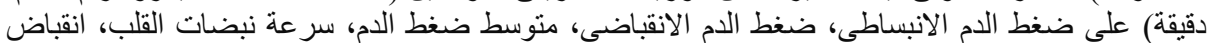

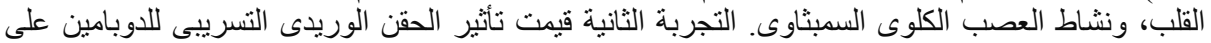

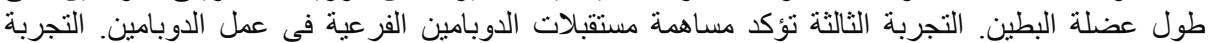

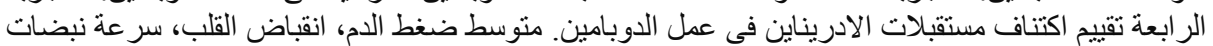

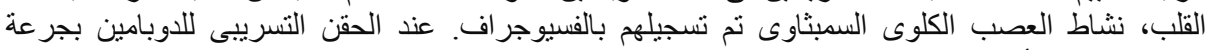

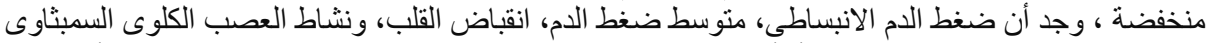

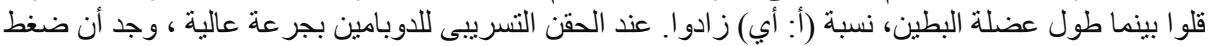

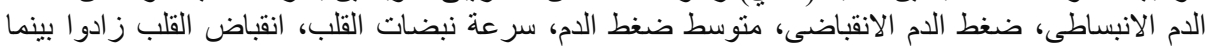

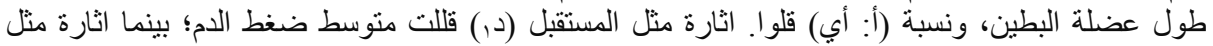

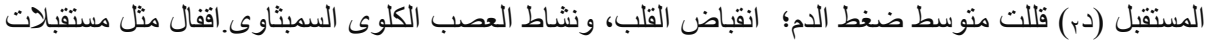

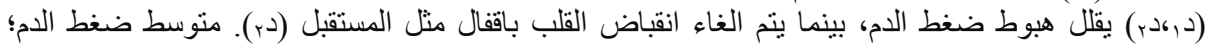

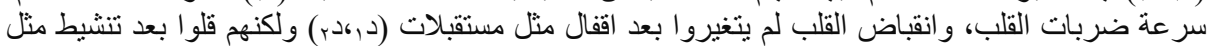

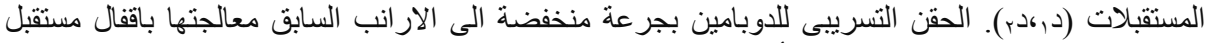

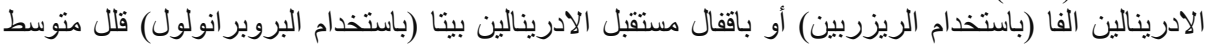

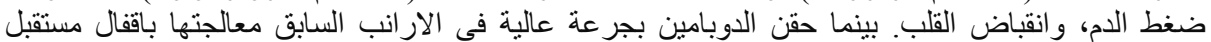

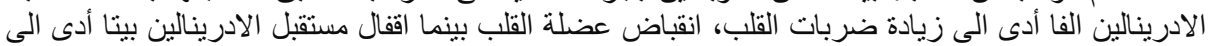

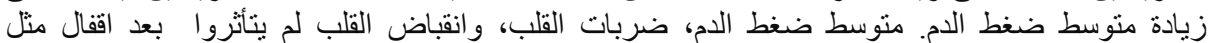

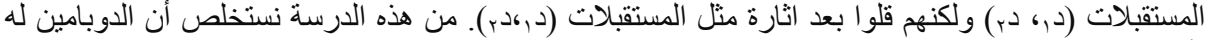

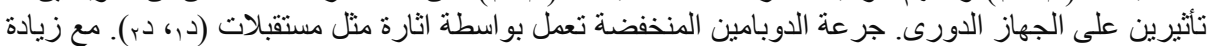

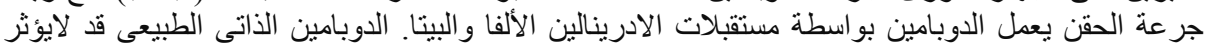
على وظائف الجهاز الدورى الاساسية. 